\title{
Operation of a H4RG-10 in the NASA Goddard Astrophyiscs Divison IR detector lab testbed
}

Gregory Mosby, Bernard J. Rauscher, Alexander Kutyrev

Gregory Mosby, Bernard J. Rauscher, Alexander Kutyrev, "Operation of a H4RG-10 in the NASA Goddard Astrophyiscs Divison IR detector lab testbed," Proc. SPIE 10709, High Energy, Optical, and Infrared Detectors for Astronomy VIII, 1070929 (6 July 2018); doi: 10.1117/12.2313503

Event: SPIE Astronomical Telescopes + Instrumentation, 2018, Austin, Texas, United States 


\title{
Operation of a H4RG-10 in the NASA Goddard Astrophyiscs Divison IR detector lab testbed
}

\author{
Gregory Mosby, Jr. ${ }^{\text {a }}$, Bernard J. Rauscher ${ }^{\mathrm{a}}$, and Alexander Kutyreva, b \\ ${ }^{a}$ NASA Goddard Space Flight Center, 8800 Greenbelt Rd, Greenbelt, MD, U.S.A. \\ ${ }^{\mathrm{b}}$ University of Maryland - College Park, College Park, MD , U.S.A.
}

\begin{abstract}
Future space and ground based missions in the near infrared are planning to or will utilize the next generation of Teledyne's HxRG detectors, the $\mathrm{HgCdTe} 4 \mathrm{~K} x \mathrm{~K}$ array (H4RG). The science cases of such missions will require optimal stability and noise performance. To assess the detailed performance of the H4RG, we have developed a small single detector testbed in NASA Goddard's Astrophysics Division IR detector lab. The testbed operates a H4RG array inside a large dewar using a room temperature Leach controller. The dewar will include two integrating spheres with controlled apertures using NIR LEDs as light sources as well as a calibrated photodiode to precisely measure flux. We present preliminary results of a banded H4RG-10 array on the bench. In the near future, we plan to use the test bed to investigate the specific origins of electronic noise in the test bed, persistence, and other flux dependent nonlinearities.
\end{abstract}

Keywords: near infrared detectors, HgCdTe, photodiodes

\section{INTRODUCTION}

Near infrared detectors provide access to a broad range of astrophysical features and phenomena unavailable in shorter wave bands. These include viewing the earliest stages of star and planet formation to observing the earliest stages of galaxy formation at high redshift. The current generation of near infrared sensors have taken advantage of the improved production and scaling of hybridized HgCdTe photodiode arrays. Teledyne's H2RGs (2048 x 2048 pixel arrays) are being used in 3 instruments of the James Webb Space Telescope ${ }^{1,2}$ as well as Hubble's WFC $3 .^{3}$ In addition, numerous ground based observatories world-wide utilize these arrays, e.g in MMIRS of the Multiple Mirror Telescope and Magellan observatories, ${ }^{4}$ Keck's MOSFIRE, ${ }^{5}$ EMIR of the Gran Telescopio Canarias, ${ }^{6}$ and RSS-NIR of the Southern African Large Telescope. ${ }^{7}$

The newest near infrared focal plane arrays from Teledyne, the H4RG-10, have been developed for the Wide Field InfraRed Space Telescope (WFIRST) and represent a natural evolution to higher resolution (to a $4096 \mathrm{x}$ 4096 pixel array) and performance from the H2RG. ${ }^{8,9}$ However, there are some key differences. The pixel size of the H4RG-10 is 10 microns instead of 18 microns which requires overcoming the technical challenges laid out in Ref. 9. Pixel pitch differences aside, we expect it to be necessary to optimize and calibrate these new arrays in a similar fashion as we have calibrated previous generation arrays to correct for detector peculiarities. These peculiarities include interpixel capacitance corrections, ${ }^{10}$ flux dependent nonlinearity also known as reciprocity failure, ${ }^{11}$ persistence, ${ }^{12,13}$ and more recently the brighter-fatter effect. ${ }^{14}$ Characterizing these effects and obtaining an accurate model for them and how they affect the noise properties of a sensor will be necessary to optimize future missions that use these arrays.

To examine the characteristics of $\mathrm{HgCdTe}$ arrays with the specific goals of developing a detailed physical model of these photodiode arrays and their response to light, we have designed a H4RG test bed. The test bed includes an H4RG-10 on a 3 axis stage, a pair of integrating spheres to provide uniform Lambertian illumination, and optics to image a sub-pixel spot along the array as well as optics to image a side port of the test dewar. We detail the design of the test bed in Sec. 2. We summarize the current status of the system in Sec. 3. We list a few of the planned tests and conclude in Sec. 4 and 5, respectively. With a flexible test bed to probe detector

Further author information: (Send correspondence to Gregory Mosby)

Gregory Mosby: E-mail: gregory.mosby@nasa.gov, Telephone: 13012862935

High Energy, Optical, and Infrared Detectors for Astronomy VIII, edited by Andrew D. Holland, James Beletic, Proc. of SPIE Vol. 10709, 1070929 - (c) 2018 SPIE CCC code: $0277-786 \mathrm{X} / 18 / \$ 18 \cdot$ doi: $10.1117 / 12.2313503$

Proc. of SPIE Vol. 10709 1070929-1 
performance under controlled conditions, we aim to identify a physical model for an array's response to light and this impact on its measurement abilities (noise). Lowering the instrumental systematics associated with detector properties will be essential in accomplishing the ambitious mission goals of the future.

\section{DESIGN OF DETECTOR TEST BED}

The detector test bed we've designed re-purposes a large aluminum dewar from previous space flight projects. The large dewar is outfitted with several existing electrical, cooling, and vacuum pump ports. We utilize these existing ports to provide input and output signals to the H4RG array using a Leach controller, cool the detector using a cryocooler, and pump down the system. Figure 1 shows a picture of the test dewar with the lid open using a pair of gas springs.

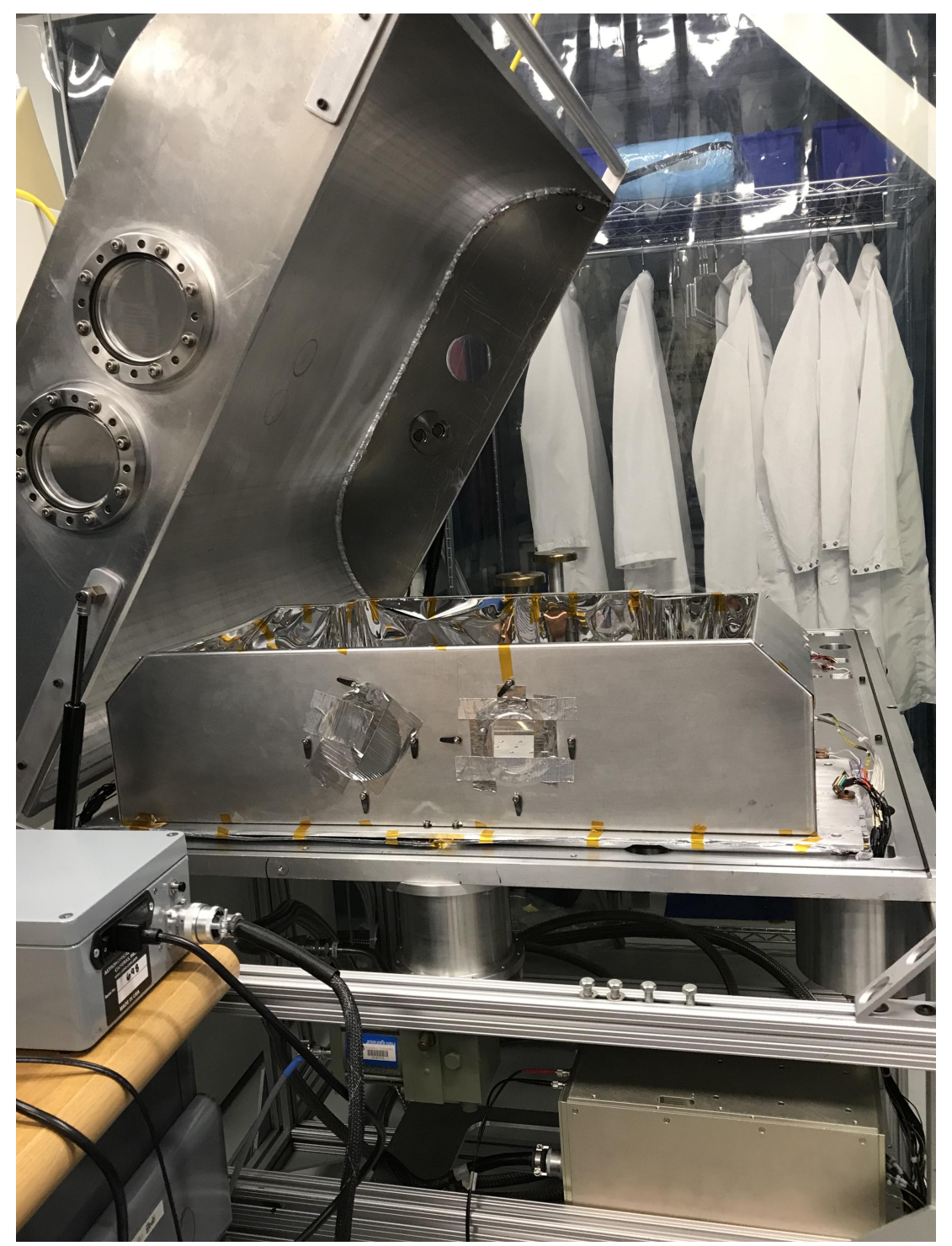

Figure 1. Image of the test dewar with the lid in the open position. The test dewar contains a smaller internal chamber seen on the near side of the dewar to which the cyrocooler's stages are coupled. Beneath the dewar the cryocooler (left) and Leach controller (right) are seen connected via their respective ports. The Leach's power supply is also seen in the foreground on the lab bench. The vacuum pump port is parallel and on the opposite side of the dewar from the Leach port. 
Though the dewar's largest internal volume is under vacuum, the cooling stages of the cryocooler are coupled to a smaller volume within the dewar we will call the internal chamber. The internal chamber is where the measurement and majority of the testing will take place. The primary tests we aim to perform require measuring the detector's response to illumination. We design our test bed to provide a uniform illumination using near infrared LEDs mounted to a dual integrating sphere. In addition to the detector's response to flat illumination, we would also like the ability to probe possible intra-pixel sensitivities and take test images through the test dewar side port. To perform these tests, we add additional optics on-axis to the detector to either image a small spot on the detector plane or image the side port. Figure 2 shows a CAD model of the design planned inside the internal chamber. The internal chamber will house the detector on a 3-axis stage (Sec. 2.1), the LED light source and integrating spheres (Sec. 2.2) to generate a uniform illumination and any necessary optics (Sec. 2.3).

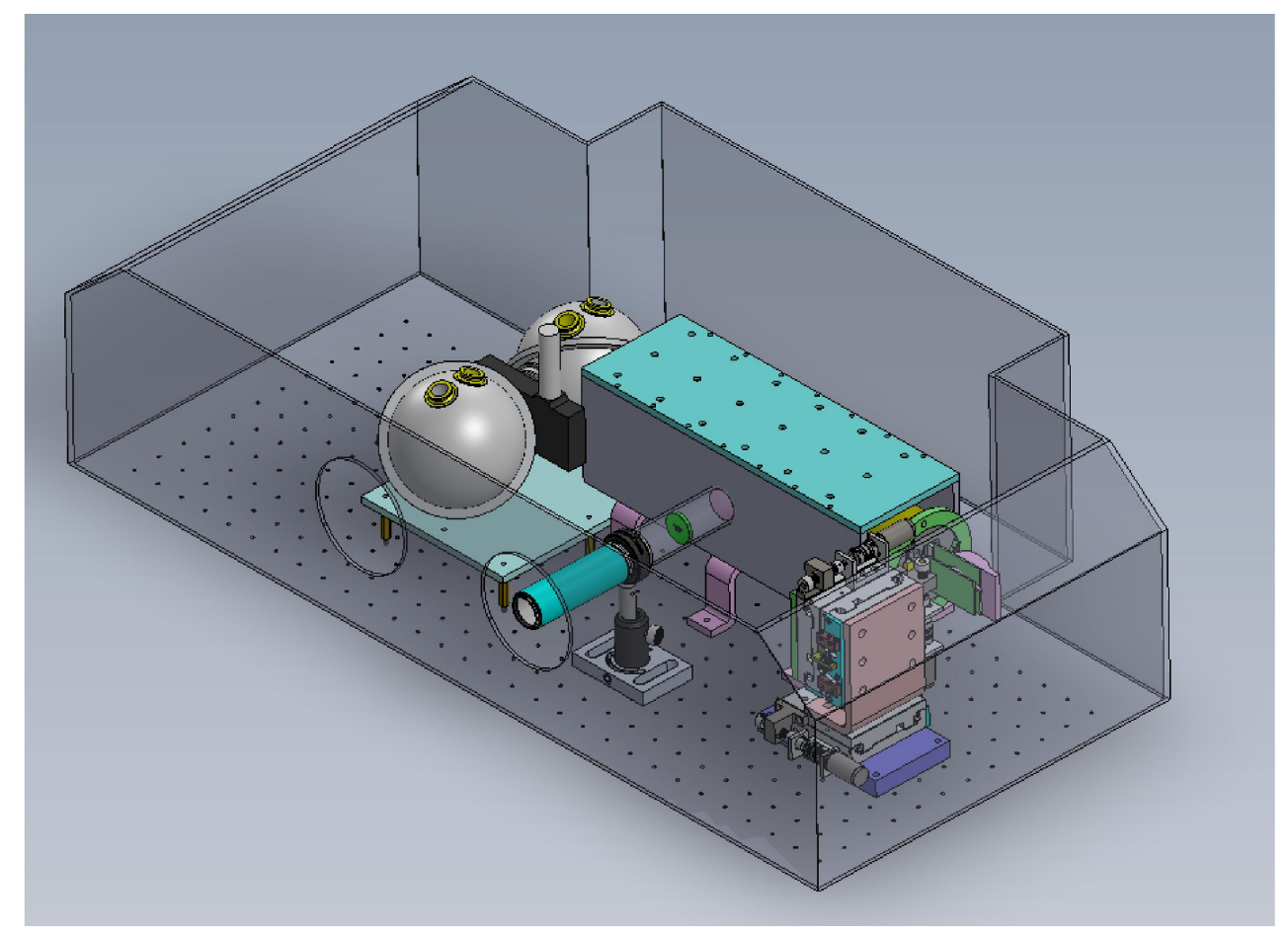

Figure 2. CAD model of the internal chamber of the test dewar. The internal chamber will house the detector on a 3 axis stage, a pair of integrating spheres to provide a uniform light source, and additional optics to provide imaging of a sub-pixel spot on the detector plane or an image of the side port of the test dewar.

\subsection{Detector}

The H4RG-10 is a recent generation of $\mathrm{HgCdTe}$ photodiode arrays from Teledyne containing 16 million pixels in a $4096 \times 4096$ array with the rectangular ring of 4 pixels formatted as reference pixels as with previous generations (see Fig. 3). The H4RG-10 we use is a spare "banded-array" detector from the WFIRST collaboration with main components of the pixel designed varied across its area and with a cutoff wavelength of 2.5 microns. This will conveniently allow for comparison tests of the different pixel designs.

The H4RG is controlled and read out using a single Leach controller with 4 8-channel video boards (ARC-46) that interface with the host computer through a fiber PCIe card. The signals from the Leach to the detector are carried by a suite of 6 cables ( 3 analog cables, 3 digital cables), using stainless steel coaxial cable for video signals inside the dewar and phosphor bronze twisted pairs for digital signals inside the dewar. For the first test images, we have used the Owl acquisition software, but we have begun moving to using a Python based acquisition and control software being developed by Lane Meier in the Detector Characterization Lab at NASA Goddard. 

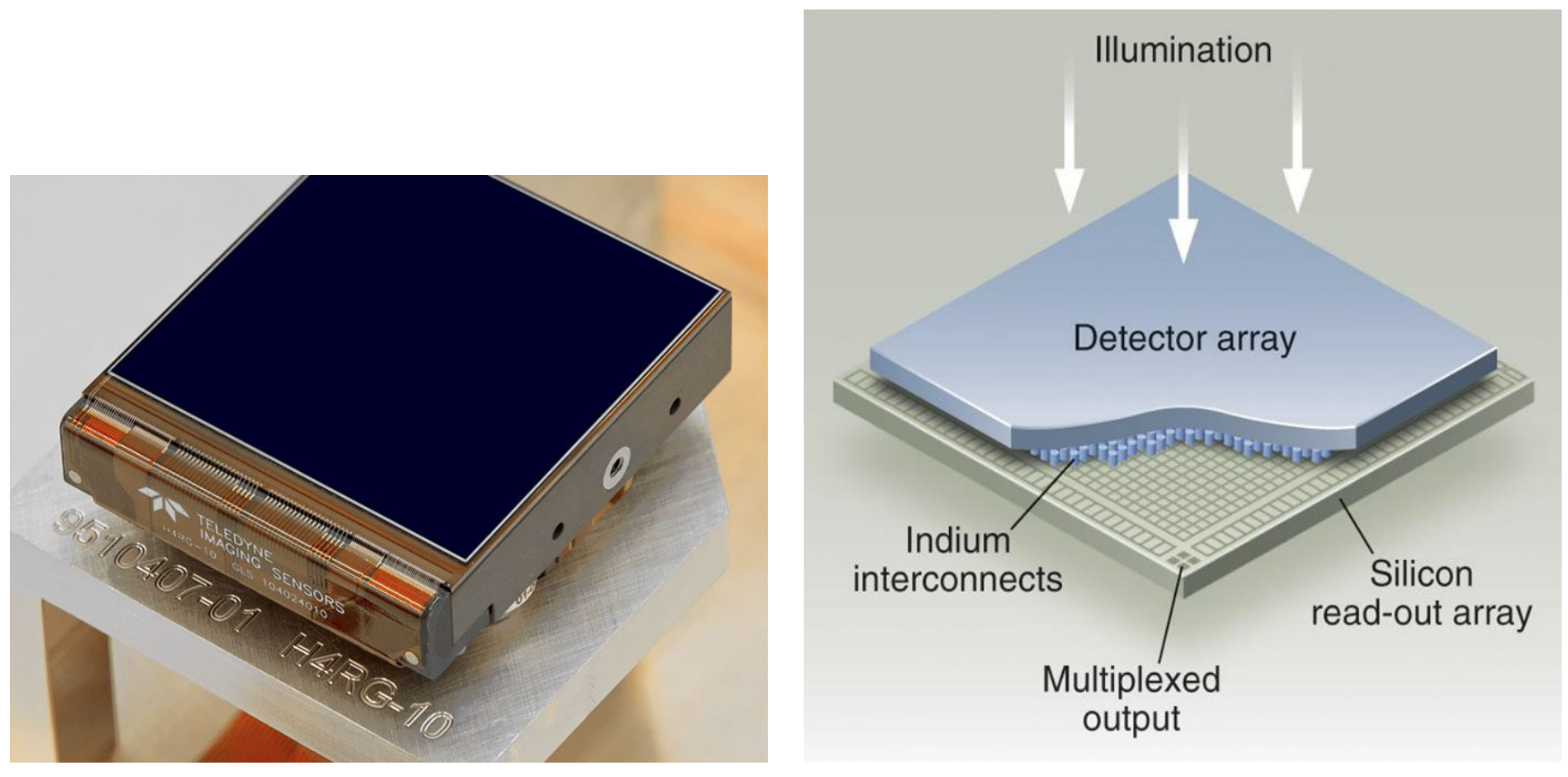

Figure 3. Left: Image of H4RG array from Teledyne website. Right: Cartoon of HgCdTe array on silicon multiplexer from Ref. 9.

\subsection{Integrating spheres}

The integrating spheres will provide illumination of the array with NIR light. A NIR led input on one sphere will provide the primary illumination. The input flux from the LED will be diffused from the first integrating sphere into a second integrating sphere where the light will be allowed to exit through a small port. The output flux of the two sphere system will be controlled by a sliding shutter between the adjoining ports of the two spheres. This will allow for variable fluxes to be obtained. To control the stability of the output of the LED, we plan to build a closed loop monitor to maintain constant current and output of the near infrared LED. Though integrating spheres produce uniform illumination at the plane of their exit ports, as the distance from the exit port increases, the uniformity decreases. In our system, we have decided on a integrating sphere distance that provide $>90 \%$ uniformity and provides radiation similar to an $\mathrm{f} / 7.9$ beam in the absence of other optics as would be expected from the WFIRST camera.

\subsection{Optics}

We use a simple combination of lens, mirrors and filters in our setup. The spot optics in the test setup are arranged to provide an on-axis sub 10 micron spot on the detector array. The optics image a minified image of a 25 micron sized pinhole located at the exit port of the second integrating sphere at the array. The optics are held at fixed distance and normal to the detector plane in a baffled and sealed $\mathrm{U}$ channel to minimize scattered light. The side port optics are designed to provide an image of one of the windowed ports of the vacuum chamber when a mirror is in place. With the $\mathrm{U}$ channel design, we can switch between either the uniform illumination (no optics), spot illumination, or side port imaging mode by simply switching out the top of the U channel.

\section{CURRENT STATUS}

After obtaining a spare WFIRST banded array, the detector harness was tested and verified with a safe-to-mate procedure, and the H4RG-10 was successfully powered and operated at room temperature on the bench. At room temperature on the bench all outputs were understandably saturated, but we performed a difference between two subsequent reads to form a correlated double sample (Fig. 4). The 32 output channel pattern is readily seen, but the dominant features in this image are from the electronics and out of range saturated pixel values. 


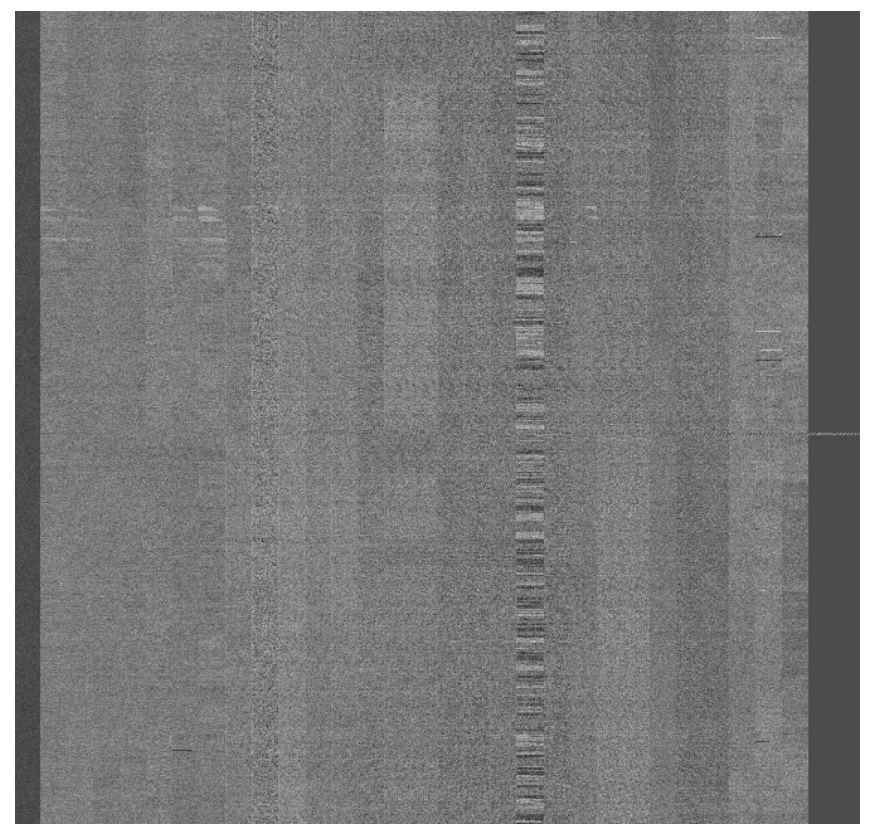

Figure 4. CDS image of the room temperature test of the H4RG-10. The difference of two subsequent mostly saturated images taken with the H4RG-10 on the bench shows mostly noise from the room temperature and unoptimized electronics.

The dewar vacuum and cooling systems have been tested. The vacuum performance is plotted in Fig. 5 . The test dewar, though large, reached an intermediate vacuum of about $5.5 \times 10^{-4} \mathrm{mBar}$. After the first pump down, the dewar was wiped down and cleaned to possibly improve this. The cryocooler was turned on to verify functionality and a more detailed cool down is planned.

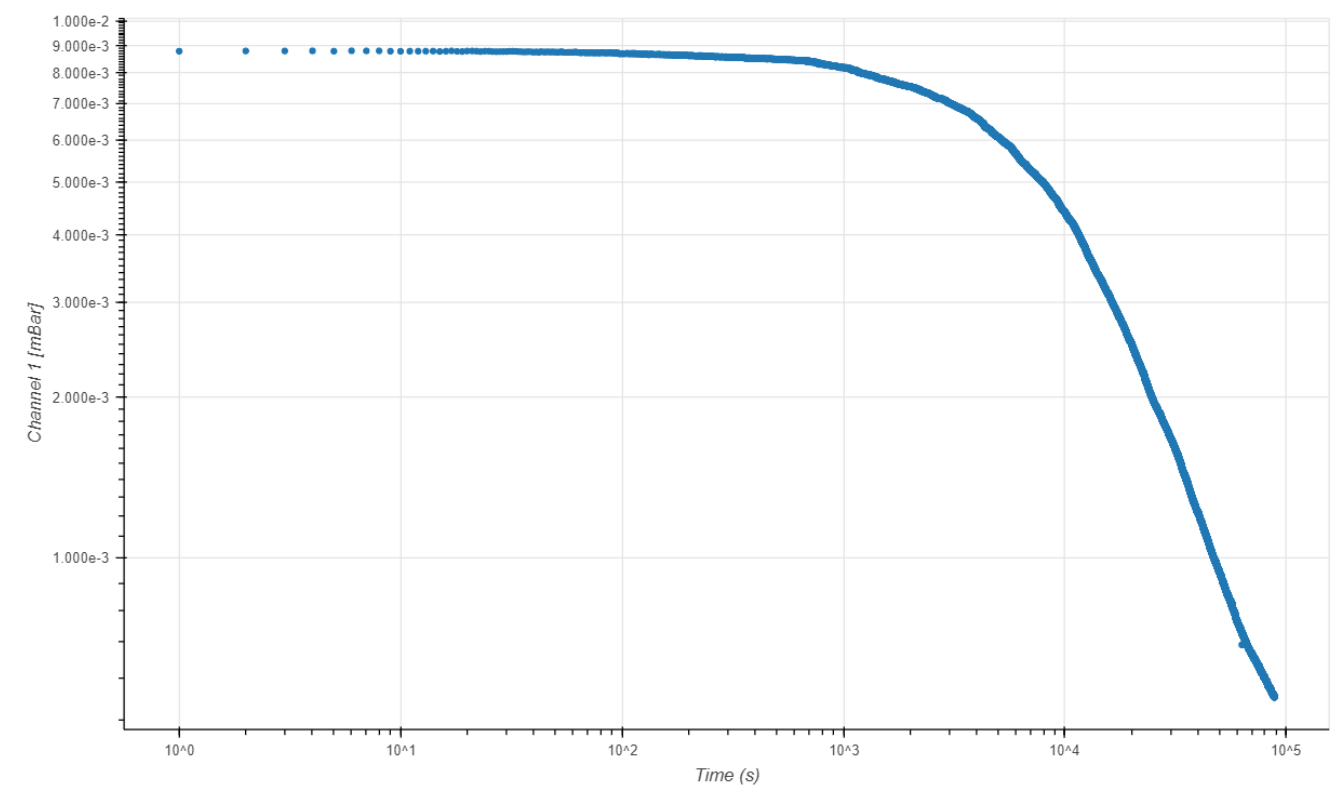

Figure 5. Logarithmic plot of preliminary vacuum performance. Vacuum tests show that our test dewar is capable of a soft, or intermediate, vacuum $\sim 5.5 \times 10^{-4}$ mBar. The test dewar has been cleaned with the aim to improve future pump downs. 
The next major step in operating the test bed will be cooling the detector and verifying its cold operation in the internal chamber of the test dewar. The detector mount and stage components for the internal chamber have been designed and are in fabrication. We hope to have cold operation of the H4RG-10 by the end of this summer.

\section{FUTURE TESTS}

The goal of creating this test bed is to provide flexible and detailed study of an H4RG-10 and its response to light with the aim of developing a detailed physical model that can be used to optimize this latest generation of $\mathrm{HgCdTe}$ photodiode arrays to lower systematics and noise in their measurements. Thus, the first tests with the test bed planned are a series of exposures varying flux. These data will allow sensitive measurements of reciprocity failure and persistence and the relationship between the two. These data will also supplement the work began in Ref. 15 that found a new way to potentially calibrate infrared arrays using the Legendre polynomials as an orthogonal basis. This first data set will be useful in teasing out the dependence of different Legendre coefficients on detector characteristics. The second generation of tests will use the optics to look at the response of the detector pixels on a point source. This data will help assess interpixel capacitance and the brighter fatter effect.

\section{CONCLUSIONS}

The widespread use of sensitive near infrared focal plane arrays has allowed for previously time consuming and low resolution observational studies to be done quickly and with higher resolution. However, near infrared arrays present unique challenges in their calibration and often nonlinear response to illumination. If we want to maximize the science output using these arrays in future missions, we must get a tighter grasp and physical model on these peculiarities and how they impact signal measurement. To tackle this challenge, we are developing a test bed for a H4RG-10 banded array to study this detector's response to light in detail. We have operated the detector on the bench, tested the vacuum and cooling systems of the test dewar and await a detector mount to begin testing the array's cold operation. By studying this banded array in detail, we hope to move closer to a complete model of how these arrays respond to light and how detector characteristics affect this.

\section{ACKNOWLEDGMENTS}

This work was made possible by the NASA Postdoctoral Program Fellowship and NASA IRAD funds. The authors would also like to acknowledge the help and support of the WFIRST project, specifically Roger Foltz and Lane Meier in the Goddard Detector Characterization Lab. In addition, the authors would like to acknowledge the help of graduate student Joe Durbak for his help in the vacuum testing.

\section{REFERENCES}

[1] James D. Garnett, Mark C. Farris, S. S. W. M. Z. D. N. H. S. J. G. L. S. P. D. D. S. F. E. F. S. M., "2kx2k molecular beam epitaxy hgcdte detectors for the james webb space telescope nircam instrument," (2004).

[2] Rauscher, B. J., Boehm, N., Cagiano, S., Delo, G. S., Foltz, R., Greenhouse, M. A., Hickey, M., Hill, R. J., Kan, E., Lindler, D., Mott, D. B., Waczynski, A., and Wen, Y., "New and better detectors for the jwst near-infrared spectrograph," Publications of the Astronomical Society of the Pacific 126(942), 739 (2014).

[3] S. M. Baggett, J. W. MacKenty, R. A. K. T. B. B. H. S. D. V. K.-P. K. S. L. A. R. R. G. R. J. H. J. K., "Wfc3 detectors: on-orbit performance," (2010).

[4] Brian A. McLeod, Daniel Fabricant, J. G. P. M. G. N. R. E. S. S. E. H. E., "Mmt and magellan infrared spectrograph," (2004).

[5] Kulas, K. R., McLean, I. S., and Steidel, C. C., "Performance of the HgCdTe detector for MOSFIRE, an imager and multi-object spectrometer for Keck Observatory," in [High Energy, Optical, and Infrared Detectors for Astronomy V], 8453, 84531S (July 2012).

[6] Francisco Garzon, David Abreu, S. B. S. C. J. J. D. A. B. F. F. J. F. F. G. C. G. P. L. A. M. J. P. J. P. P. R. R. R. V. S. A. V., "Emir: the gtc nir multi-object imager-spectrograph," (2004). 
[7] Wolf, M. J., Mulligan, M. P., Smith, M. P., Adler, D. P., Bartosz, C. M., Bershady, M. A., Buckley, D. A. H., Burse, M. P., Chordia, P. A., Clemens, J. C., Epps, H. W., Garot, K., Indahl, B. L., Jaehnig, K. P., Koch, R. J., Mason, W. P., Mosby, G., Nordsieck, K. H., Percival, J. W., Punnadi, S., Ramaprakash, A. N., Schier, J. A., Sheinis, A. I., Smee, S. A., Thielman, D. J., Werner, M. W., Williams, T. B., and Wong, J. P., "Project status of the Robert Stobie spectrograph near infrared instrument (RSS-NIR) for SALT," in [Ground-based and Airborne Instrumentation for Astronomy V], 9147, 91470B (July 2014).

[8] Content, D., Aaron, K., Abplanalp, L., Anderson, K., Capps, R., Chang, Z., Dooley, J., Egerman, R., Goullioud, R., Klein, D., Kruk, J., Kuan, G., Melton, M., Ruffa, J., Underhill, M., and Van Buren, D., "Wide-Field InfraRed Survey Telescope (WFIRST) 2.4-meter mission study," in [UV/Optical/IR Space Telescopes and Instruments: Innovative Technologies and Concepts VI], 8860, 88600E (Sept. 2013).

[9] Eric C. Piquette, William McLevige, J. A. A. W., "Progress in development of h4rg-10 infrared focal plane arrays for wfirst-afta," (2014).

[10] Moore, A. C., Ninkov, Z., and Forrest, W. J., "Quantum efficiency overestimation and deterministic crosstalk resulting from interpixel capacitance," Optical Engineering 45(7), 076402-076402-9 (2006).

[11] Biesiadzinski, T., Lorenzon, W., Newman, R., Schubnell, M., Tarl, G., and Weaverdyck, C., "Reciprocity failure in hgcdte detectors: Measurements and mitigation," Publications of the Astronomical Society of the Pacific 123(906), 958 (2011).

[12] Smith, R. M., Zavodny, M., Rahmer, G., and Bonati, M., "A theory for image persistence in HgCdTe photodiodes," in [High Energy, Optical, and Infrared Detectors for Astronomy III], 7021, 70210J (July 2008).

[13] Mosby, G., Eggen, N., Wolf, M., Jaehnig, K., and Kotulla, R., "Persistence characterization and data calibration scheme for the RSS-NIR H2RG detector on SALT," in [High Energy, Optical, and Infrared Detectors for Astronomy VII], 9915, 99152U (July 2016).

[14] Plazas, A. A., Shapiro, C., Smith, R., Huff, E., and Rhodes, J., "Laboratory measurement of the brighterfatter effect in an h2rg infrared detector," Publications of the Astronomical Society of the Pacific 130(988), 065004 (2018).

[15] Rauscher, B., "WFIRST: Principal Components Analysis of H4RG-10 Near-IR Detector Data Cubes," in [American Astronomical Society Meeting Abstracts \#231], American Astronomical Society Meeting Abstracts 231, 152.28 (Jan. 2018). 